\title{
Optimal image restoration with the fractional Fourier transform
}

\author{
M. Alper Kutay and Haldun M. Ozaktas \\ Department of Electrical Engineering, Bilkent University, 06533 Bilkent, Ankara, Turkey
}

Received August 29, 1997; accepted September 29, 1997; revised manuscript received November 7, 1997

The classical Wiener filter, which can be implemented in $O(N \log N)$ time, is suited best for space-invariant degradation models and space-invariant signal and noise characteristics. For space-varying degradations and nonstationary processes, however, the optimal linear estimate requires $O\left(N^{2}\right)$ time for implementation. Optimal filtering in fractional Fourier domains permits reduction of the error compared with ordinary Fourier domain Wiener filtering for certain types of degradation and noise while requiring only $O(N \log N)$ implementation time. The amount of reduction in error depends on the signal and noise statistics as well as on the degradation model. The largest improvements are typically obtained for chirplike degradations and noise, but other types of degradation and noise may also benefit substantially from the method (e.g., nonconstant velocity motion blur and degradation by inhomegeneous atmospheric turbulence). In any event, these reductions are achieved at no additional cost. (C) 1998 Optical Society of America [S0740-3232(98)00604-8]

OCIS codes: $070.2590,100.3020$.

\section{INTRODUCTION}

Restoration of degraded or distorted and noisy images is a basic problem in image and optical processing, with many applications. The objective is to reduce or eliminate the degradations or distortions that are introduced typically by the transmission channel and the sensing environment. A variety of approaches to degradation removal have been proposed (for instance, see Ref. 1). The effectiveness of these methods depends on the observation model and the design criteria used as well as on the prior knowledge available about the desired signal, degradation process, and noise. One of the most popular observation models is of the form

$$
\mathbf{o}=\mathscr{H}(\mathbf{f})+\mathbf{n},
$$

where $\mathbf{o}$ is the observed signal, $\mathbf{f}$ is the signal we wish to recover, $\mathbf{n}$ is an additive and possibly nonstationary noise signal, and $\mathscr{H}$ is the system representing undesired linear time-varying distortion. A frequently used design criterion is the mean-square error (MSE), and we usually consider a linear estimation of the form

$$
\hat{\mathbf{f}}=\mathscr{G}(\mathbf{o}) \text {. }
$$

Then the problem is to find the operator $\mathscr{G}_{\mathrm{opt}}$ that minimizes the MSE.

The well-known classical Wiener filtering presents a solution to the above problem under the assumption that the signals involved are stationary and $\mathscr{H}$ is a timeinvariant system. This filter turns out to be a timeinvariant one that corresponds to a multiplicative filter in the Fourier domain and thus can be implemented in $O(N \log N)$ time, where $N$ is the space-bandwidth product of the images, i.e., the number of pixels in the image. The general solution when the above assumptions do not hold is also known. ${ }^{2}$ However, since the resulting linear operator is not time-invariant and thus cannot be expressed as a convolution, obtaining this most general lin- ear estimate requires computational time of $O\left(N^{2}\right)$ as opposed to $O(N \log N)$ for the time-invariant case. We can still seek the optimal ordinary Fourier domain Wiener filter, but this filter is not as satisfactory as the general linear estimator.

The possibility of realizing various time-varying operations by filtering in fractional Fourier domains was suggested in Ref. 3. An exact analytical solution for the optimal filtering problem (analogous to the Wiener filtering problem) in fractional domains for one-dimensional (1D) signals was given in Ref. 4. Filtering in a fractional Fourier domain can be implemented as efficiently as filtering in the conventional Fourier domain, since the fractional Fourier transformation has a fast digital algorithm ${ }^{4,5}$ and can also be optically realized much like the usual Fourier transform. $^{6-10}$ Thus any improvement obtained with the fractional Fourier transformation comes at no additional cost.

In this paper the concept of filtering in fractional Fourier domains is applied to the problem of estimating images [or other two-dimensional (2D) signals] with spacevarying statistics in the presence of space-varying degradation and noise. Expressions for the 2D optimal filter function in fractional domains will be given for transform domains characterized by the two-order parameters of the $2 \mathrm{D}$ fractional Fourier transform. Then we will seek the optimal values of these parameters, thus achieving the smallest possible error with the proposed method. Since the class of fractional Fourier domain filters is a subclass of the class of all linear operators, for the arbitrary time-varying degradation model the MSE obtained by the proposed method will still not be as small as the one obtained by the general linear estimator. However, the class of proposed filters is a much broader class than ordinary Fourier domain filters, and it is possible to obtain smaller MSE's in comparison with ordinary Fourier domain filters. It will be shown in the ex- 
amples that the method is very effective, especially when the noise and degradations are of chirped nature. It will also be shown that substantial reduction in error can be achieved for other interesting types of degradation and noise that are encountered in practice. We expect the proposed method to be applicable to other degradationand-noise models not discussed in this paper.

\section{TWO-DIMENSIONAL FRACTIONAL FOURIER TRANSFORMATION}

In this section we give the definition of the $2 \mathrm{D}$ fractional Fourier transformation. The fractional Fourier transform is the generalization of the ordinary Fourier transform. ${ }^{6,11-14}$ The fractional Fourier transform has been found to have several applications in fields including the solution of differential equations, quantum mechanics, diffraction theory and optical propagation, optical systems and signal processing, swept-frequency filters, space-variant filtering and multiplexing, and the study of time- and space-frequency distributions. ${ }^{3,6,9,12-24}$ Its analog optical implementation was discussed in Refs. 6, 9, and 15 , and its digital implementation was discussed in Ref. 5.

One of the most important properties of the fractional Fourier transform is its relation to time- and spacefrequency representations. , $^{9,25,26}$ This property states that the fractional Fourier transformation corresponds to a rotation in the time- and space-frequency plane for certain members of Cohen's class. It leads us to the concept of fractional Fourier domains ${ }^{27}$ and also suggests a way of performing certain time-varying operations by employing the fractional Fourier transform. ${ }^{3,4}$

The $a$ th-order fractional Fourier transform of a 1D function $f(x)$ may be defined for $0<|a|<2$ as

$$
\begin{aligned}
{\left[\mathscr{F}^{a}(f)\right](x)=} & f_{a}(x) \\
= & \int B_{a}\left(x, x^{\prime}\right) f\left(x^{\prime}\right) \mathrm{d} x^{\prime}, \\
B_{a}\left(x, x^{\prime}\right)= & A_{\phi} \exp \left[i \pi \left(x^{2} \cot \phi\right.\right. \\
& \left.\left.-2 x x^{\prime} \csc \phi+x^{\prime 2} \cot \phi\right)\right], \\
A_{\phi}= & (|\sin \phi|)^{-1 / 2} \exp [i \pi \operatorname{sgn}(\sin \phi) / 4-i \phi / 2],
\end{aligned}
$$

where $\phi \equiv a \pi / 2$. The kernel $B_{a}\left(x, x^{\prime}\right)$ approaches $\delta(x$ $\left.-x^{\prime}\right)$ or $\delta\left(x+x^{\prime}\right)$ when $a$ approaches 0 or \pm 2 , respectively. The definition is easily extended outside the interval $[-2,2]$ since $\mathscr{F}^{4}$ is the identity operation.

A direct generalization of the above definition to $2 \mathrm{D}$ signals is given by

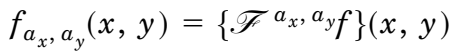

$$
\begin{aligned}
& =\iint B_{a_{x}, a_{y}}\left(x, y ; x^{\prime}, y^{\prime}\right) f\left(x^{\prime}, y^{\prime}\right) \mathrm{d} x^{\prime} \mathrm{d} y^{\prime}, \\
& B_{a_{x}, a_{y}}\left(x, y ; x^{\prime}, y^{\prime}\right) \\
& =B_{a_{x}}\left(x, x^{\prime}\right) B_{a_{y}}\left(y, y^{\prime}\right) .
\end{aligned}
$$

The 2D transform kernel is the product of two 1D kernels as in the case of the ordinary Fourier transform, but we allow different orders $a_{x}$ and $a_{y}$ for the two coordinates. Efficient analog optical implementations of such anamorphic $2 \mathrm{D}$ transforms has been demonstrated. ${ }^{10,28,29}$ Digital computation is also possible with direct modifications to the algorithm developed for $1 \mathrm{D}$ signals ${ }^{5}$ since $2 \mathrm{D}$ transformation is defined as separable so that the associated kernel is just the product of two 1D kernels.

\section{FILTERING IN FRACTIONAL FOURIER DOMAINS}

In this section the mathematical definition of the problem is given, and our approach to its solution is formulated. The solution for the case of a linear space-invariant degradation model with stationary processes is the wellknown optimal Wiener filter, which can be implemented efficiently with the fast Fourier transform. For spacevarying degradation models and nonstationary signals and noise, the optimal recovery operator is also known but in general requires $O\left(N^{2}\right)$ time for implementation, where $N$ is the space-bandwidth product of the signal, i.e., the number of pixels in the image.

First, we briefly review the general linear filtering problem. Our signal observation model can be written as

$$
o(x, y)=\iint h\left(x, y ; x^{\prime}, y^{\prime}\right) f\left(x^{\prime}, y^{\prime}\right) \mathrm{d} x^{\prime} \mathrm{d} y^{\prime}+n(x, y),
$$

where $h\left(x, y ; x^{\prime}, y^{\prime}\right)$ is the kernel of the degradation model and $n(x, y)$ is the additive noise term. (All integrals are from minus infinity to plus infinity unless otherwise stated.) We assume that as prior knowledge we know the correlation functions $R_{f f}\left(x, y ; x^{\prime}, y^{\prime}\right)$ $=E\left[f(x, y) f^{*}\left(x^{\prime}, y^{\prime}\right)\right], \quad R_{n n}\left(x, y ; x^{\prime}, y^{\prime}\right)=E[n(x, y)$ $\left.\times n^{*}\left(x^{\prime}, y^{\prime}\right)\right]$ of the input signal (desired signal) $\mathbf{f}$ and the noise. We further assume that the noise is independent of the input $\mathbf{f}$ and is zero mean, i.e., $E[n(x, y)]$ $=0$ for all $x$ and $y$, and that we know the degradation model. Under these assumptions we can also find the cross-correlation function $R_{f o}\left(x, y ; x^{\prime}, y^{\prime}\right)=E[f(x, y)$ $\left.\times o^{*}\left(x^{\prime}, y^{\prime}\right)\right]$ of the processes $\mathbf{f}$ and $\mathbf{o}$ and the correlation function $R_{o o}\left(x, y ; x^{\prime}, y^{\prime}\right)=E\left[o(x, y) o^{*}\left(x^{\prime}, y^{\prime}\right)\right]$ by using Eq. (4).

Consider the most general linear estimate of the form

$$
\hat{f}(x, y)=\iint g\left(x, y ; x^{\prime}, y^{\prime}\right) f\left(x^{\prime}, y^{\prime}\right) \mathrm{d} x^{\prime} \mathrm{d} y^{\prime} .
$$

Our design criteria is the MSE, which is defined as

$$
\sigma_{e}{ }^{2}=E\left(\|\mathbf{f}-\hat{\mathbf{f}}\|^{2}\right),
$$

where $E(\cdot)$ denotes the expectation operator and $\|\cdot\|$ denotes the norm

$$
\|\mathbf{f}\|^{2}=\iint|f(x, y)|^{2} \mathrm{~d} x \mathrm{~d} y .
$$

This definition [Eq. (6)] of the MSE with the norm defined in Eq. (7) is appropriate for nonstationary signals whose functional representations are square integrable (of finite energy). [For stationary processes, the MSE may be defined as the expected value of the magnitude squared of the difference term. ${ }^{2}$ ] The problem is then to find the optimal recovery operator kernel, denoted by 


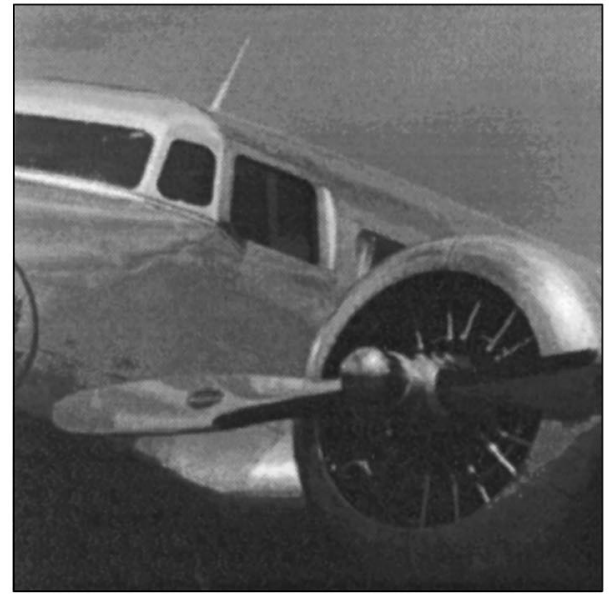

(a)

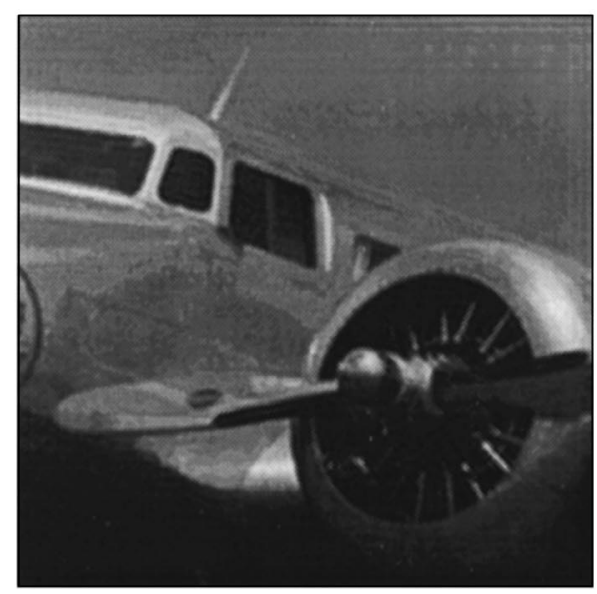

(c)

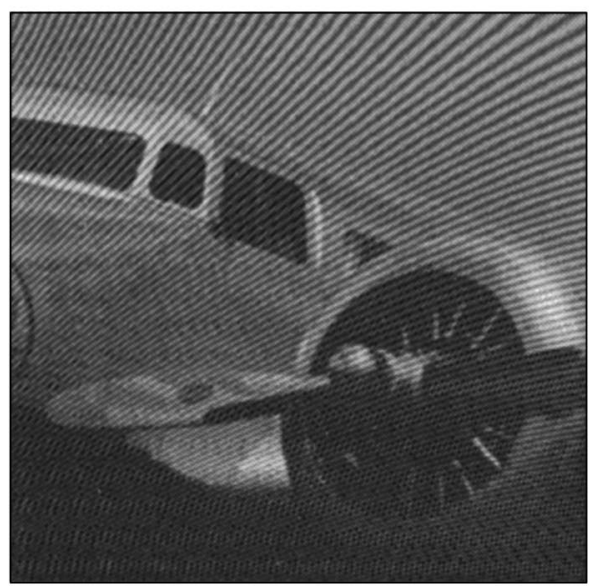

(b)

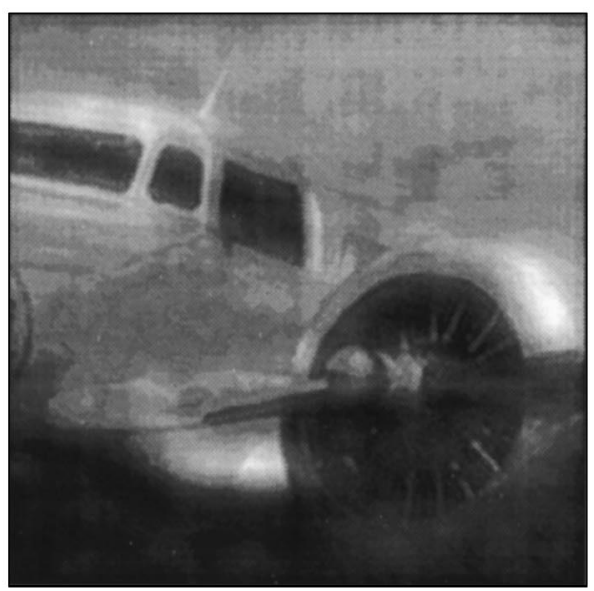

(d)

Fig. 1. (a) Original (desired) plane image; (b) corrupted image (SNR $\approx 1$ ), (c) estimated image obtained by filtering in optimum fractional Fourier domain $\left(a_{x}=0.4, a_{y}=-0.6\right)$, (d) image restored by filtering in ordinary Fourier domain.

$g_{\text {opt }}\left(x, y ; x^{\prime}, y^{\prime}\right)$, which minimizes the MSE. The solution to this problem, with the linear estimate defined in Eq. (5), is known, and $g_{\text {opt }}\left(x, y ; x^{\prime}, y^{\prime}\right)$ is the kernel that satisfies the following equation ${ }^{2}$ :

$$
\begin{aligned}
& R_{f o}\left(x, y ; x^{\prime}, y^{\prime}\right) \\
& \quad=\iint g_{\text {opt }}\left(x, y ; x^{\prime \prime}, y^{\prime \prime}\right) R_{o o}\left(x^{\prime \prime}, y^{\prime \prime} ; x^{\prime}, y^{\prime}\right) \mathrm{d} x^{\prime \prime} \mathrm{d} y^{\prime \prime}
\end{aligned}
$$

Equation (8) can be solved numerically to yield the kernel of the optimal linear recovery operator. However, application of this estimation operator [see Eq. (5)] on a given distorted and noisy signal would require $O\left(N^{2}\right)$ time, where $N$ is the space-bandwidth product of the signals (the number of pixels for images). In this paper we restrict our estimate so that it corresponds to a multiplication by a filter function in the fractional Fourier domain. This estimate can be written as

$$
\hat{f}(x, y)=\mathscr{F}^{-a_{x},-a_{y}}\left\{m(x, y) \mathscr{F}^{a_{x}, a_{y}}[o(x, y)]\right\},
$$

where $\mathscr{F}^{a_{x}, a_{y}}$ is the 2D fractional Fourier transformation operator with different-order parameters for each dimension ${ }^{10}$ and $m(x, y)$ is the multiplicative filter. According to Eq. (9), we first take the 2D fractional Fourier transform of the observed signal $o(x, y)$ with orders $a_{x}$ and $a_{y}$ and then multiply the transformed signal with the filter $m(x, y)$ and take the inverse 2D fractional Fourier transform of the resulting signal. Thus the filter $m(x, y)$ has been applied in the fractional Fourier domain of or$\operatorname{ders} a_{x}$ and $a_{y}$. We note that for $a_{x}=a_{y}=1$ this estimate corresponds to filtering in the conventional Fourier domain. With this form of estimation operator the minimization problem considered in this paper is to find the optimal filter function, denoted by $m_{\text {opt }}(x, y)$, that minimizes the MSE defined in Eq. (6) with the estimate $\hat{f}(x, y)$ given by Eq. (9). The class of fractional Fourier domain filters is a subclass of the class of all linear operators, so the linear filter we find is not the most optimal among all linear operators. However, it is a much 
broader class than (time-invariant) Fourier domain filters, and in many problems involving time-varying degradation models and nonstationary processes, it is possible to obtain smaller MSE's in comparison with filtering in the conventional Fourier domain. This reduction in MSE comes at no additional cost, because the resulting filter can be implemented digitally in $O(N \log N)$ time just like the ordinary Fourier transform, ${ }^{5}$ or can be implemented optically with the same kind of hardware as that of the ordinary Fourier transform. ${ }^{6-9}$

We finally note that although beyond the scope of the present paper, various extensions and refinements of the classical filtering problem (for instance, see Refs. 34-36 and the references therein) can also be applied to the fractional Fourier domain filtering problem that we have considered.

\section{OPTIMAL FRACTIONAL FOURIER DOMAIN FILTER}

The solutions of the $2 \mathrm{D}$ and $1 \mathrm{D}$ problems are similar. Our estimate is given by which is nothing but the well-known orthogonality condition. $^{30,31}$ The above equation states that the best linear MSE $\hat{f}_{a_{x}, a_{y}}(x, y)$ is an orthogonal projection of the signal $f_{a_{x}, a_{y}}(x, y)$ onto the space of observations.

The optimum filter function $m_{\text {opt }}(\cdot, \cdot$ ) can be solved from Eq. (11) by use of the definition of $\hat{f}_{a_{x}, a_{y}}(x, y)$,

$$
m_{\mathrm{opt}}(x, y)=\frac{R_{f_{a_{x}, a_{y}, o a_{x}, a_{y}}}(x, y ; x, y)}{R_{o_{a_{x}, a_{y}, o a_{x}, a_{y}}}(x, y ; x, y)},
$$

where

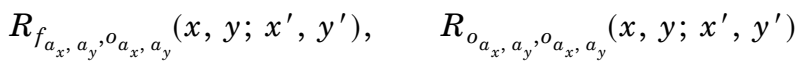

are the correlation functions in the transform domain $\left(a_{x}, a_{y}\right)$. These correlation functions can easily be calculated from the correlation functions in the spatial domain so that the filter function is given by

$$
m_{\mathrm{opt}}(x, y)=\frac{\iiint \int B_{a_{x}, a_{y}}\left(x, y ; x^{\prime}, y^{\prime}\right) B_{-a_{x},-a_{y}}\left(x, y ; x^{\prime \prime}, y^{\prime \prime}\right) R_{f, o}\left(x^{\prime}, y^{\prime} ; x^{\prime \prime}, y^{\prime \prime}\right) \mathrm{d} x^{\prime} \mathrm{d} y^{\prime} \mathrm{d} x^{\prime \prime} \mathrm{d} y^{\prime \prime}}{\iiint \int B_{a_{x}, a_{y}}\left(x, y ; x^{\prime}, y^{\prime}\right) B_{-a_{x},-a_{y}}\left(x, y ; x^{\prime \prime}, y^{\prime \prime}\right) R_{o, o}\left(x^{\prime}, y^{\prime} ; x^{\prime \prime}, y^{\prime \prime}\right) \mathrm{d} x^{\prime} \mathrm{d} y^{\prime} \mathrm{d} x^{\prime \prime} \mathrm{d} y^{\prime \prime}} .
$$

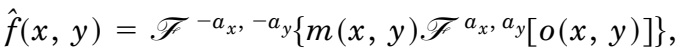

$$
\begin{aligned}
& =\iint B_{-a_{x},-a_{y}}\left(x, y ; x^{\prime \prime}, y^{\prime \prime}\right) m\left(x^{\prime \prime}, y^{\prime \prime}\right) \\
& \times \iint B_{a_{x}, a_{y}}\left(x^{\prime \prime}, y^{\prime \prime} ; x^{\prime}, y^{\prime}\right) o\left(x^{\prime}, y^{\prime}\right) \\
& \times \mathrm{d} x^{\prime} \mathrm{d} y^{\prime} \mathrm{d} x^{\prime \prime} \mathrm{d} y^{\prime \prime},
\end{aligned}
$$

and the error is

$$
\begin{aligned}
& \sigma_{e}^{2}=E\left(\|\mathbf{f}-\hat{\mathbf{f}}\|^{2}\right) \\
& =E\left(\| f(x, y)-\mathscr{F}^{\left.-a_{x},-a_{y}\left\{m(x, y) \mathscr{F}^{a_{x}}, a_{y}[o(x, y)]\right\} \|^{2}\right) .}\right.
\end{aligned}
$$

Since the 2D fractional Fourier transformation is unitary, this MSE is equal to the error in the transform domain:

$$
\begin{aligned}
\sigma_{e}^{2} & =E\left(\left\|\mathbf{f}_{a_{x}, a_{y}}-\hat{\mathbf{f}}_{a_{x}, a_{y}}\right\|^{2}\right) \\
& =E\left[\left\|f_{a_{x}, a_{y}}(x, y)-m(x, y) o_{a_{x}, a_{y}}(x, y)\right\|^{2}\right] .
\end{aligned}
$$

For particular values of $a_{x}$ and $a_{y}$, the optimal filter function that minimizes the above error can be shown to satisfy the following equation:

$$
E\left\{\left[f_{a_{x}, a_{y}}(x, y)-\hat{f}_{a_{x}, a_{y}}(x, y)\right] o_{a_{x}, a_{y}}^{*}(x, y)\right\}=0,
$$

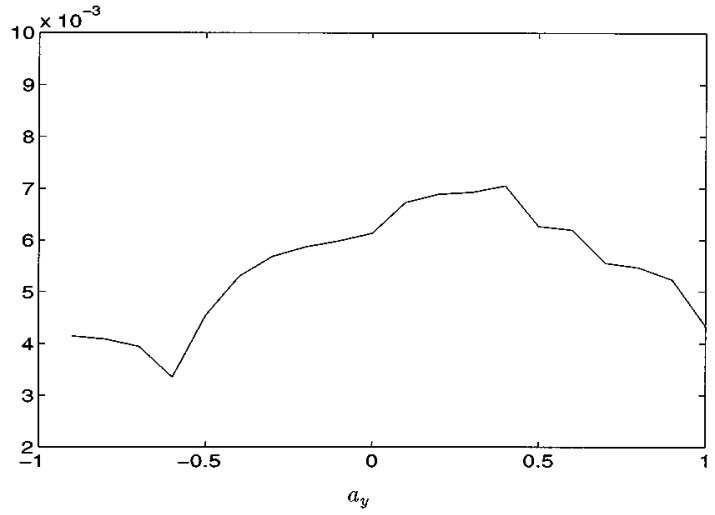

(a)

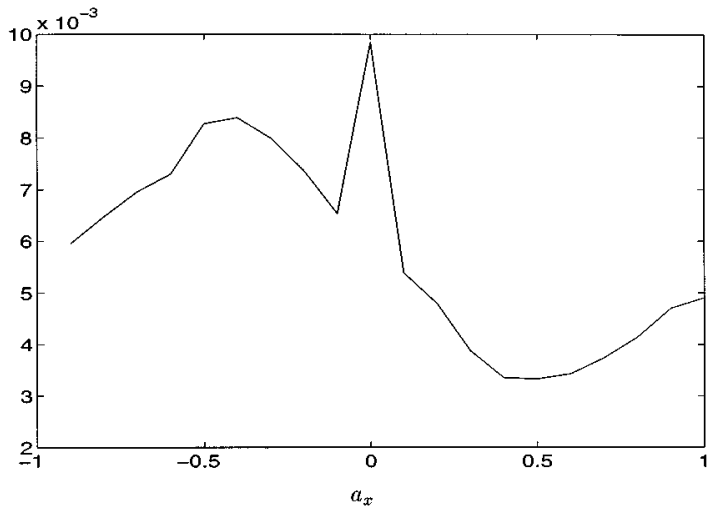

(b)

Fig. 2. (a) MSE versus $a_{y}$ for $a_{x}=0.4$, (b) MSE versus $a_{x}$ for $a_{y}=-0.6$. 


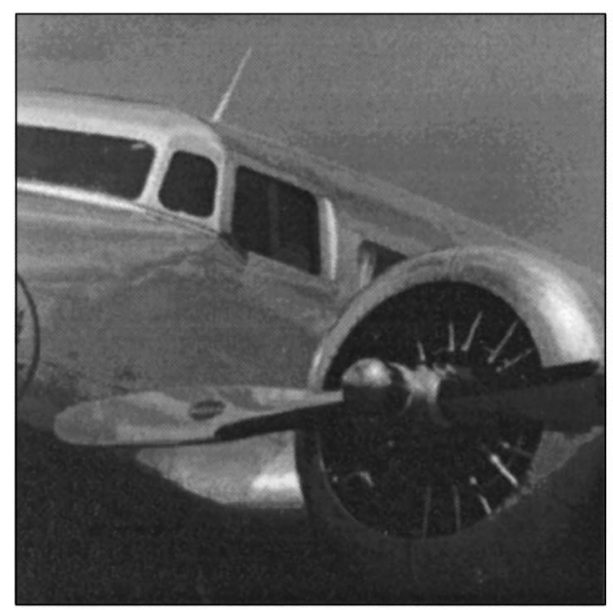

(a)

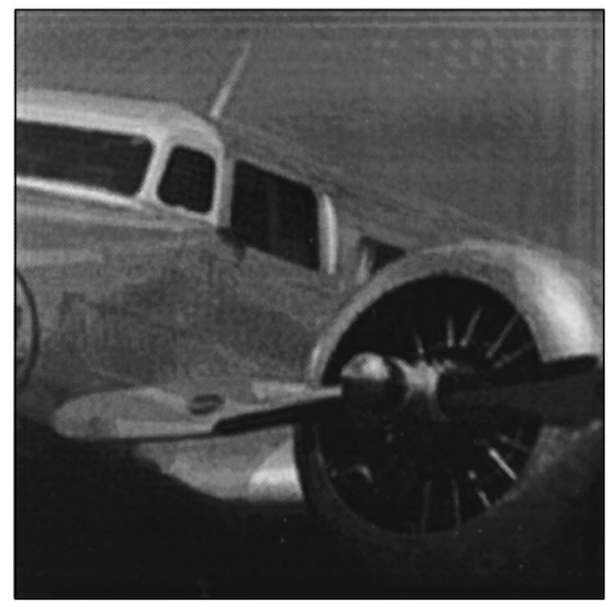

(c)

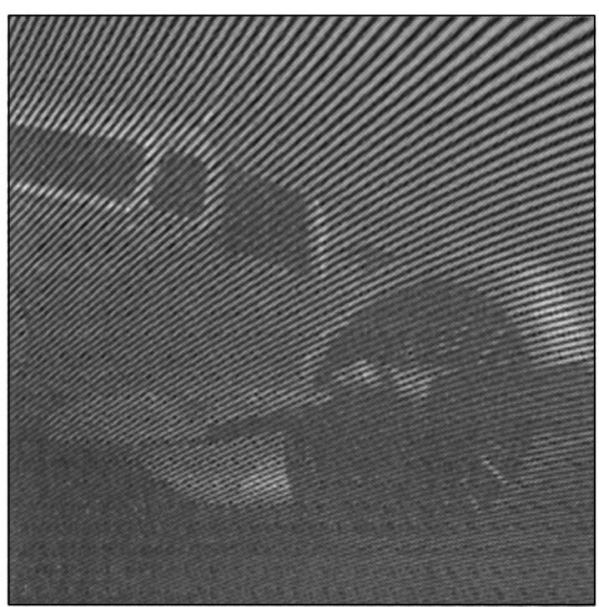

(b)

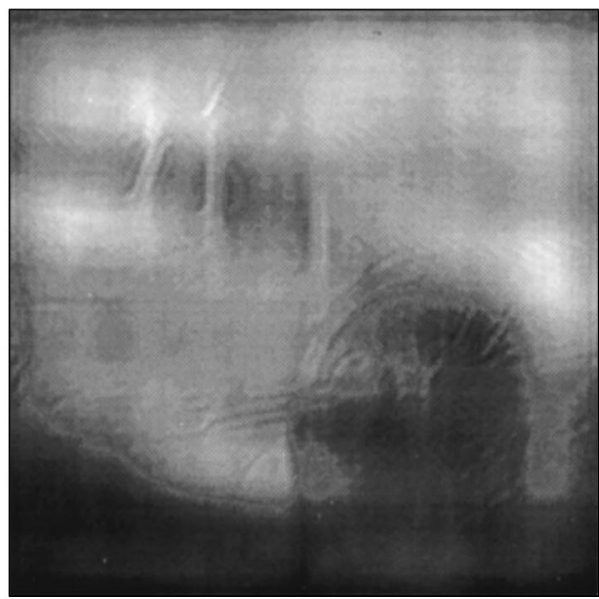

(d)

Fig. 3. (a) Original (desired) plane image, (b) corrupted image (SNR $\approx 0.1$ ), (c) estimated image obtained by filtering in optimum fractional Fourier domain $\left(a_{x}=0.4, a_{y}=-0.6\right)$, (d) image restored by filtering in ordinary Fourier domain.

Equation (12) provides us the optimal multiplicative filter function in the fractional domain defined by the parameters $a_{x}, a_{y}$. To find the optimal values of $a_{x}$ and $a_{y}$-that is, the domain in which the smallest error is obtained-we plug the optimum filter function into the MSE expression,

$$
\begin{aligned}
& \sigma_{e, o}{ }^{2}=E\left\{\iint\left[f_{a_{x}, a_{y}}(x, y)-\hat{f}_{a_{x}, a_{y}}(x, y)\right]\right. \\
& \left.\times\left[f_{a_{x}, a_{y}}(x, y)-\hat{f}_{a_{x}, a_{y}}(x, y)\right]^{*} \mathrm{~d} x \mathrm{~d} y\right\}
\end{aligned}
$$

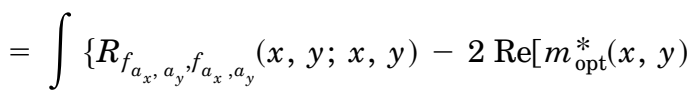

$$
\begin{aligned}
& \left.\times R_{f_{a_{x}, a_{y}, o_{a_{x}, a_{y}}}}(x, y ; x, y)\right] \\
& \left.+\left|m_{\text {opt }}(x, y)\right|^{2} R_{o_{a_{x}, a_{y}, o}, a_{y}}(x, y ; x, y)\right\} \mathrm{d} x \mathrm{~d} y,
\end{aligned}
$$

and then choose the values of $a_{x} \in[-11]$ and $a_{y}$ $\in[-11]$ that minimize $\sigma_{e, o}{ }^{2}$. (Note that MSE is peri- odic with respect to $a_{x}$ and $a_{y}$ with period 2.) These values may be found analytically in certain special cases. But these cases are exceptional. In general, we can find the optimal values of $a_{x}$ and $a_{y}$ numerically by simply calculating the MSE for sufficiently closely spaced discrete values of $a_{x}$ and $a_{y}$ (for example, with a step size of 0.1 ) and choosing the values that minimize the MSE. We can also find the optimal values by employing a standard multivariate optimization routine. ${ }^{32}$

Overall, the procedure can be outlined as follows: Given the autocorrelation functions of the input (f) and noise $(n)$ processes, along with the degradation $(\mathscr{H})$, we can find the correlation function between the input and output (o) processes and the autocorrelation function of the output process. Then, using these, we can find the optimal filter function in the fractional domain characterized by $a_{x}$ and $a_{y}$ by using Eq. (12). The optimal choices of $a_{x}$ and $a_{y}$ are then those that minimize Eq. (13). Once these are determined for the given signal and noise statistics and for distortion model, implementation of the fractional Fourier domain filter requires $O(N \log N)$ time for an image with $N$ pixels. It is important to emphasize 
that both the digital computation of the fractional Fourier transform and its optical implementation are nearly as efficient as the ordinary Fourier transform, so that the improvements in performance come at essentially no cost.

\section{EXAMPLES}

In this section we apply our method to degraded images to illustrate the applications and performance of fractional Fourier domain filtering. In the first two examples we apply the method to images corrupted by chirplike noises and show that the method is very effective and permits significant reduction in error in comparison with ordinary Fourier domain filtering for this kind of degradation. The last two examples show the performance of the method for other types of degradation, particularly for two different space-varying blur models with additive white Gaussian noise. The reduction is less spectacular for these examples.

Figure 1(a) shows the original image used. In Fig. 1(b) this image has been corrupted by the presence of two chirp waveforms with amplitudes selected so that the noise energy is comparable with that of the image, mak-

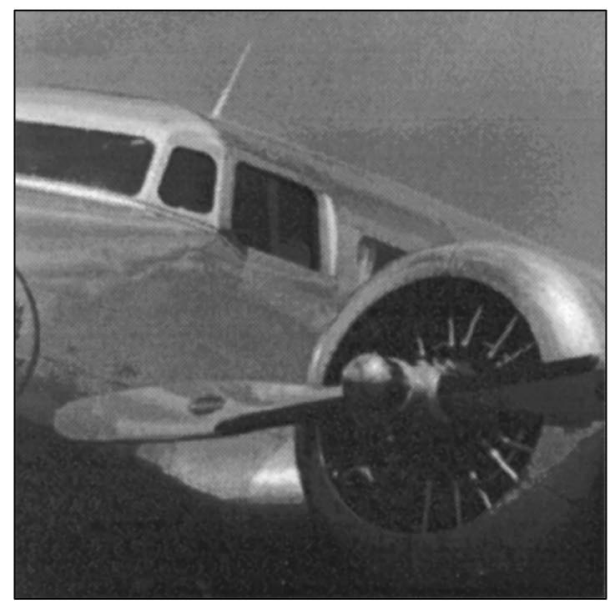

(a)

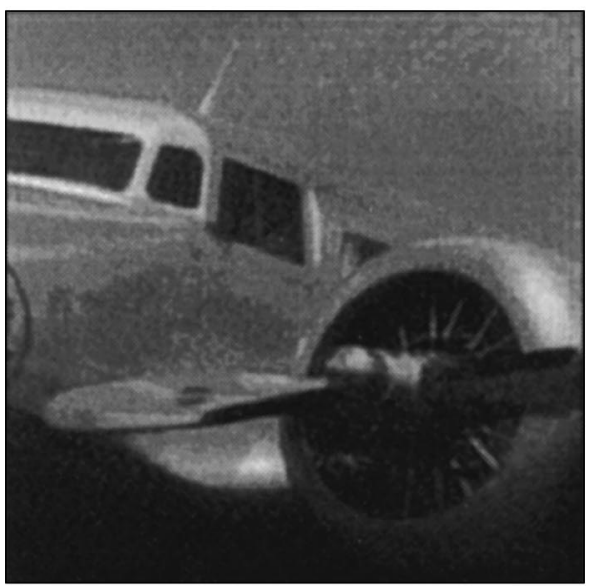

(c) ing the signal-to-noise ratio approximately one. The optimally estimated image is shown in Fig. 1(c), for which the optimal-order parameters are found to be $a_{x}=0.4$ and $a_{y}=-0.6$. The minimum MSE is $\sim 0.003$ [in this section, MSE's are normalized by the energy of the original image $E\left(\|f\|^{2}\right)$.] For comparison, we display in Fig. 1(d) the result of optimal restoration by ordinary Fourier domain filtering (corresponding to the order parameters $a_{x}=a_{y}=1$ ), which is less satisfactory, with MSE equal to 0.035 .

We plot the profiles of the MSE along the individual order parameters $\left(a_{x}\right.$ and $a_{y}$ ) around the optimal point in Figs. 2(a) and 2(b). (We recall that MSE is periodic with respect to the parameters $a_{x}$ and $a_{y}$ with period 2.) These plots show the behavior of the MSE around the optimal point where minimum MSE is achieved.

The above example is repeated with a signal-to-noise ratio $\approx 0.1$. The corresponding images are presented in Fig. 3. The benefit obtained by use of fractional Fourier domain filtering (MSE 0.006) instead of ordinary Fourier domain filtering (MSE 0.10) is much greater for this value of signal-to-noise ratio.

In the following two examples we apply the method to

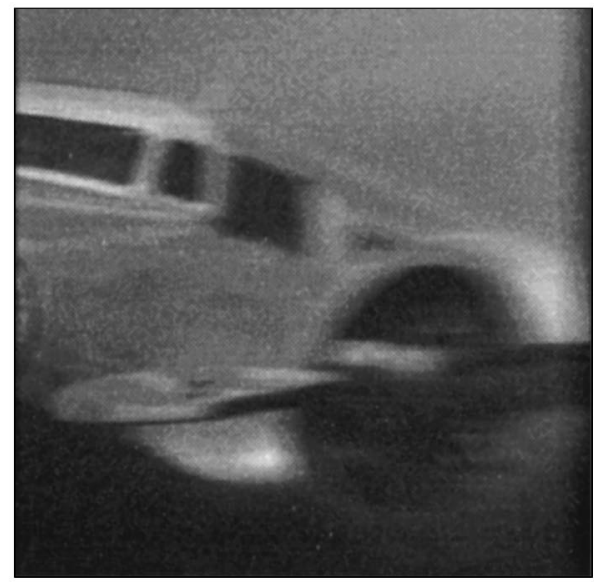

(b)

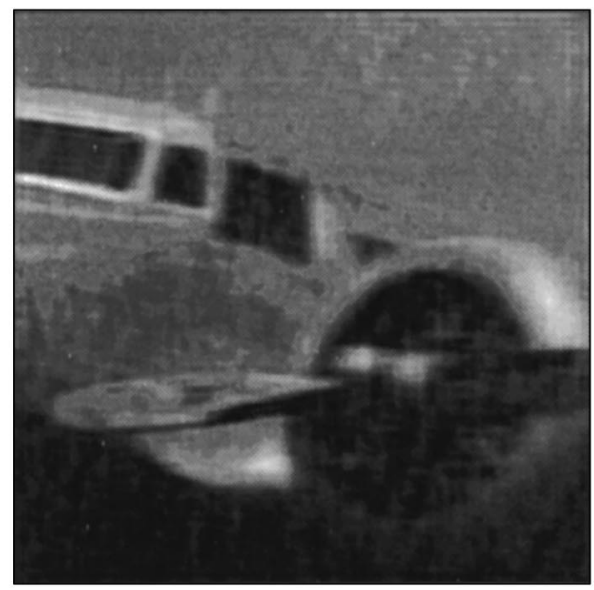

(d)

Fig. 4. (a) Original (desired) plane image, (b) degraded image, (c) estimated image obtained by filtering in optimum fractional Fourier domain $\left(a_{x}=0.7, a_{y}=0.8\right.$ ), (d) image restored by filtering in ordinary Fourier domain. 


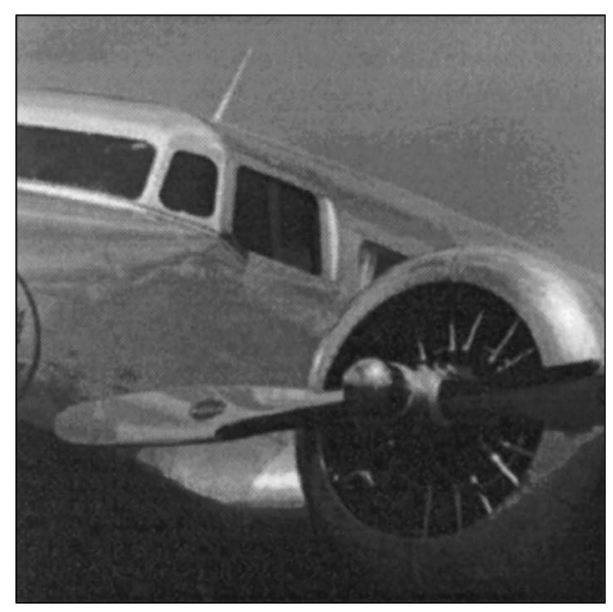

(a)

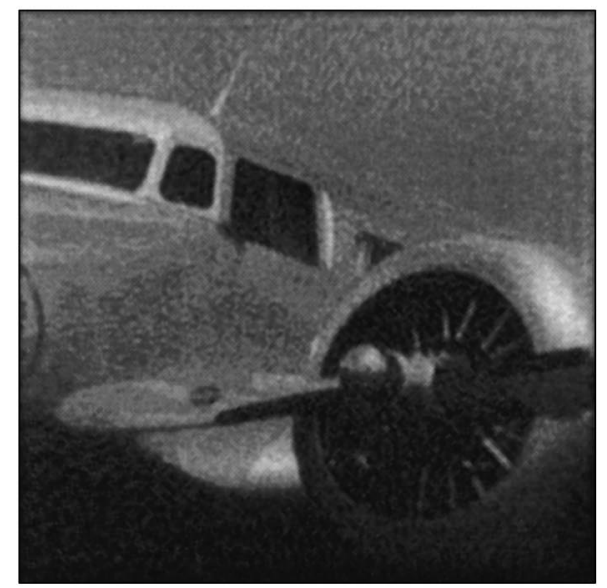

(c)

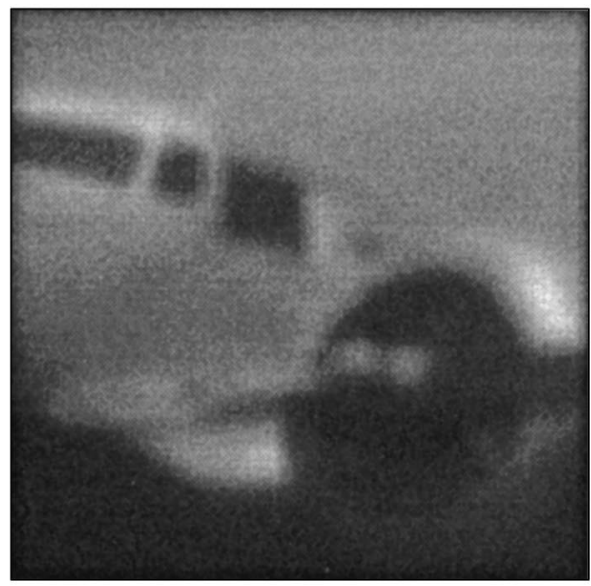

(b)

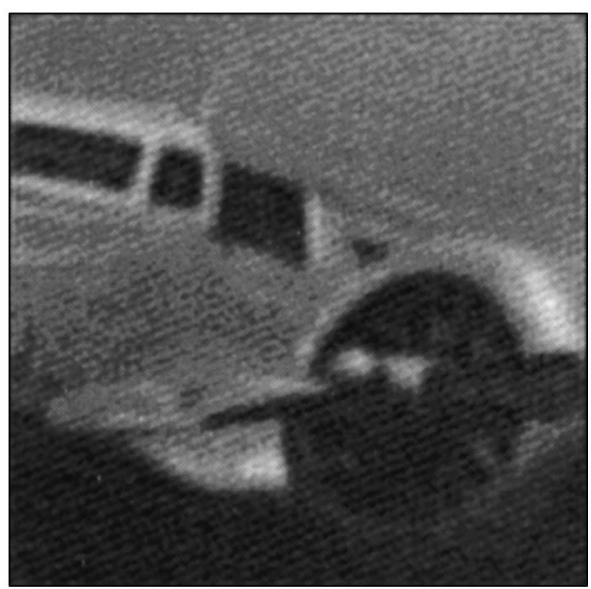

(d)

Fig. 5. (a) Original (desired) plane image, (b) degraded image, (c) estimated image obtained by filtering in optimum fractional Fourier domain $\left(a_{x}=0.4, a_{y}=0.7\right)$, (d) image restored by filtering in ordinary Fourier domain.

images degraded with different space-varying blur models together with an additive white Gaussian noise. These examples illustrate the performance of the method for degradation and noise that are not of a chirped nature.

In this example we consider nonconstant-velocity motion blur. This blur corresponds to the degradation that is the result of accelerated linear motion (in which velocity is increasing linearly) between the object and the camera during exposure. [We should note that in the case of constant-velocity motion the degradation is timeinvariant so that the optimal filtering domain turns out to be the ordinary Fourier domain $\left.\left(a_{x}=a_{y}=1\right)\right]$. For this type of degradation the kernel [see Eq. (4)] is given by

$h\left(x, y ; x^{\prime}, y^{\prime}\right)=\frac{1}{\alpha x+\alpha_{0}} \operatorname{rect}\left(\frac{x-x^{\prime}}{\alpha x+\alpha_{0}}-\frac{1}{2}\right) \delta\left(y-y^{\prime}\right)$,

where $\alpha$ and $\alpha_{0}$ are the parameters of the distortion model and correspond to acceleration and initial velocity, respectively. The additive noise is white Gaussian noise whose energy is equal to one fourth of the signal energy (input SNR of 4). Figure 4(a) shows the original (desired) image, and Fig. 4(b) shows the distorted image ( $\alpha$ $=0.01$ and $\alpha_{0}=0.3$ ). The optimally estimated image is shown in Fig. 4(c), for which the optimal order parameters are found to be $a_{x}=0.7$ and $a_{y}=0.8$. The minimum MSE is $\approx 0.0097$. For comparison we have displayed in Fig. 4(d) the result of optimal restoration using ordinary Fourier domain filtering (corresponding to the order parameters $a_{x}=a_{y}=1$ ), which is less satisfactory, with a MSE equal to 0.0382 .

In the last example we consider degradation that corresponds to space-varying atmospheric turbulence. This degradation is the result of inhomogeneous statistical properties of the turbulent media ${ }^{33}$ and occurs when an image covers several isoplanatic patches (regions where statistical properties of the turbulent media can be taken to be constant). The kernel of the degradation is given by

$$
\begin{aligned}
h\left(x, y ; x^{\prime}, y^{\prime}\right)= & \exp \left\{-\pi \alpha^{2}(x, y)\right. \\
& \left.\times\left[\left(x-x^{\prime}\right)^{2}+\left(y-y^{\prime}\right)^{2}\right]\right\},
\end{aligned}
$$


where $\alpha(x, y)$ is a function of $x$ and $y$, which makes the degradation space varying. In our example, $\alpha(x, y)$ $=\alpha_{0}+\beta(x, y)$, where $\beta(x, y)$ represents the fluctuation around $\alpha_{0}(=0.1)$ and is a slowly varying function that is obtained by low-pass filtering the white Gaussian noise. (When an image consists of a single isoplanatic patch, the function $\alpha(x, y)$ reduces to a constant $\alpha_{0}$, and in this case the degradation becomes time invariant and can be optimally eliminated in ordinary Fourier domain.) The additive noise is again white Gaussian noise whose energy is equal to half of the energy of the signal, and it takes into account the electrical noise encountered in the camera. The desired and the distorted images are shown in Figs. 5(a) and 5(b), respectively. Figures 5(c) and 5(d) show the optimally estimated image in the optimal domain $\left(a_{x}=0.4\right.$ and $\left.a_{y}=0.7\right)$ and in the conventional Fourier domain. The minimum MSE is $\sim 0.021$ for Fig. 5(c), whereas it is 0.052 for Fig. 5(d).

The above examples show that filtering with the fractional Fourier transform permits a significant reduction in MSE for chirplike degradations and at least a substantial reduction for certain other interesting types of degradation. We believe that there should exist other examples that benefit from the proposed method to varying degrees.

\section{CONCLUSIONS}

In this paper we have shown that optimal filtering in fractional Fourier domains is effective in restoring images corrupted by certain types of distortion and noise and offers significant improvement in comparison with restored images in ordinary Fourier domains. In particular, we have seen that the method is very effective in eliminating chirplike noises, and the MSE can be improved by significant factors in comparison with ordinary Fourier domain filtering. The method is also shown to be useful for other types of degradation and noise with moderate reduction in MSE. These improvements come at no additional cost.

We expect fractional Fourier domain image-restoration techniques to find broad application in optical systems. This is because the types of distortion and noise for which the greatest benefits are obtained with respect to ordinary Fourier domain filtering arise naturally in optical systems in the form of scattering from point and line defects and twin images in holography, etc. Also, the 2D filtering process described in this paper is effectively and easily implemented with optical systems.

The examples given in this paper by no means exhaust the signal and noise characteristics for which the method is beneficial. Further characterization of the strengths and limitations of the proposed method requires further research.

M. Alper Kutay can be reached by telephone: $90-312-$ 266-4307, by fax: 90-312-266-4126, and by e-mail: kutay@ee.bilkent.edu.tr.

\section{REFERENCES}

1. J. S. Lim, Two-Dimensional Signal and Image Processing (Prentice-Hall, Englewood Cliffs, N.J., 1990).
2. F. L. Lewis, Optimal Estimation (Wiley, New York, 1986).

3. H. M. Ozaktas, B. Barshan, D. Mendlovic, and L. Onural, "Convolution, filtering, and multiplexing in fractional Fourier domains and their relation to chirp and wavelet transforms," J. Opt. Soc. Am. A 11, 547-559 (1994).

4. M. A. Kutay, H. M. Ozaktas, O. Arikan, and L. Onural, "Optimal filtering in fractional Fourier domains," IEEE Trans. Signal Process. 45, 1129-1143 (1997).

5. H. M. Ozaktas, O. Arikan, M. A. Kutay, and G. Bozdaği, "Digital computation of the fractional Fourier transform," IEEE Trans. Signal Process. 44, 2141-2150 (1996).

6. H. M. Ozaktas and D. Mendlovic, "Fourier transforms of fractional order and their optical interpretation," Opt. Commun. 101, 163-169 (1993).

7. D. Mendlovic and H. M. Ozaktas, "Fractional Fourier transformations and their optical implementation: part I," J. Opt. Soc. Am. A 10, 1875-1881 (1993).

8. H. M. Ozaktas and D. Mendlovic, "Fractional Fourier transformations and their optical implementation: part II," J. Opt. Soc. Am. A 10, 2522-2531 (1993).

9. A. W. Lohmann, "Image rotation, Wigner rotation, and the fractional Fourier transform," J. Opt. Soc. Am. A 10, 21812186 (1993).

10. A. Sahin, H. M. Ozaktas, and D. Mendlovic, "Optical implementation of the two-dimensional fractional Fourier transform with different orders in two dimensions," Opt. Commun. 120, 134-138 (1995)

11. E. U. Condon, "Immersion of the Fourier transform in a continuous group of functional transformations," Proc. Natl. Acad. Sci. USA 23, 158-164 (1937).

12. V. Namias, "The fractional Fourier transform and its application in quantum mechanics," J. Inst. Math. Appl. 25, 241-245 (1980).

13. A. C. McBride and F. H. Kerr, "On Namias's fractional Fourier transform,” IMA J. Appl. Math. 39, 159-175 (1987).

14. L. M. Almeida, "The fractional Fourier transform and timefrequency representations," IEEE Trans. Signal Process. 42, 3084-3091 (1994).

15. H. M. Ozaktas and D. Mendlovic, "Fractional Fourier optics," J. Opt. Soc. Am. A 12, 743-751 (1995).

16. H. M. Ozaktas and D. Mendlovic, "Fractional Fourier transform as a tool for analyzing beam propagation and spherical mirror resonators," Opt. Lett. 19, 1678-1680 (1994).

17. P. Pellat-Finet, "Fresnel diffraction and the fractional-order Fourier transform," Opt. Lett. 19, 1388-1390 (1994).

18. P. Pellat-Finet and G. Bonnet, "Fractional-order Fourier transform and Fourier optics," Opt. Commun. 111, 141-154 (1994).

19. L. M. Bernardo and O. D. D. Soares, "Fractional Fourier transforms and optical systems," Opt. Commun. 110, 517522 (1994).

20. T. Alieva, V. Lopez, F. Agullo-Lopez, and L. B. Almeida, "The angular Fourier transform in optical propagation problems,” J. Mod. Opt. 41, 1037-1044 (1994).

21. T. Alieva and F. Agullo-Lopez, "Optical wave propagation of fractal fields," Opt. Commun. 125, 267-274 (1996).

22 . D. T. Smithey, M. Beck, M. G. Raymer, and A. Faridanil, "Measurement of the Wigner distribution and the density matrix of a light mode using optical homodyne tomography: application to squeezed states and the vacuum," Phys. Rev. Lett. 70, 1244-1247 (1993).

23. J. R. Fonollosa and C. L. Nikias, "A new positive timefrequency distribution," in Proceedings of the IEEE International Conference on Acoustic Speech and Signal Processing (Institute of Electrical and Electronics Engineers, Piscataway, N.J., 1994), pp. IV-301-IV-304.

24. J. Wood and D. T. Barry, "Radon transformation of timefrequency distributions for analysis of multicomponent signals," IEEE Trans. Signal Process. 42, 3166-3177 (1994).

25. A. W. Lohmann and B. H. Soffer, "Relationships between the Radon-Wigner and fractional Fourier transforms," J. Opt. Soc. Am. A 11, 1798-1801 (1994).

26. H. M. Ozaktas, N. Erkaya, and M. A. Kutay, "Effect of fractional Fourier transformation on time-frequency distributions belonging to the Cohen class," IEEE Signal Process. Lett. 3(2), 40-41 (1996). 
27. H. M. Ozaktas and O. Aytür, "Fractional Fourier domains," Signal Process. 46, 119-124 (1995).

28. A. Sahin, "Two-dimensional fractional Fourier transformation and its optical implementation," Master's thesis (Bilkent University, Ankara, Turkey, 1996).

29. M. F. Erden, H. M. Ozaktas, and A. Sahin, "Design of dynamically adjustable anamorphic fractional Fourier transformer," Opt. Commun. 136, 52-60 (1997).

30. B. D. O. Anderson and J. B. Moore, Optimal Filtering (Prentice-Hall, New York, 1979).

31. A. Jazwinski, Stochastic Processes and Filtering Theory (Academic, New York, 1970).

32. W. H. Press, B. P. Flannery, S. A. Teukolsky and W. T.
Wetterling, Numerical Recipes in Pascal (Cambridge U. Press, Cambridge, 1989), pp. 574-579.

33. J. H. Shapiro, "Diffraction-limited atmospheric imaging of extended objects," J. Opt. Soc. Am. 66, 469-477 (1976).

34. J. Zhang, "The mean field theory in EM procedures for blind Markov random field image restoration," IEEE Trans. Image Process. 2, 27-40 (1993).

35. M. R. Banham and A. K. Katsaggelos, "Spatially adaptive wavelet-based multiscale image restoration," IEEE Trans. Image Process. 5, 619-634 (1996).

36. M. R. Banham and A. K. Katsaggelos, "Digital image restoration," IEEE Trans. Signal Process. 14(2), 24-41 (1997). 